\title{
Parameter estimation in large dynamic paired comparison experiments
}

\author{
Mark E. Glickman \\ Boston University, USA \\ [Received June 1997. Revised November 1998]
}

\begin{abstract}
Summary. Paired comparison data in which the abilities or merits of the objects being compared may be changing over time can be modelled as a non-linear state space model. When the population of objects being compared is large, likelihood-based analyses can be too computationally cumbersome to carry out regularly. This presents a problem for rating populations of chess players and other large groups which often consist of tens of thousands of competitors. This problem is overcome through a computationally simple non-iterative algorithm for fitting a particular dynamic paired comparison model. The algorithm, which improves over the commonly used algorithm of Elo by incorporating the variability in parameter estimates, can be performed regularly even for large populations of competitors. The method is evaluated on simulated data and is applied to ranking the best chess players of all time, and to ranking the top current tennis-players.
\end{abstract}

Keywords: Approximate Bayesian estimation; Bradley-Terry model; Chess; Ranking; State space models; Tennis

\section{Introduction}

Paired comparison models address data that arise from situations in which two objects or treatments are compared at the same time to determine a degree of preference. Examples include modelling choice behaviour (preference of one soft drink to another or the preference of the Democratic presidential candidate to the Republican candidate), competitive ability in sports (determining the strengths of teams in basketball or baseball) and many others. A review of some examples and issues involved in paired comparison modelling was given by David (1988) and Bradley (1984).

Recent work (Fahrmeir and Tutz, 1994; Glickman, 1993) extended the usual (static) paired comparison models by including parameters that are time varying. These 'dynamic' paired comparison models are appropriate, for example, for modelling paired comparison data arising from competitive sports where player or team abilities change over time. When the size of the population of competitors is reasonably small, the methodologies developed in these papers present no computational difficulties. However, these methods are inadequate for large populations of competitors because the computation becomes intractable. For example, with more than 30000 chess players playing over 450000 games each year in United States Chess Federation (USCF) chess tournaments, other less computationally intensive methods for fitting dynamic paired comparison models need to be considered.

This paper presents a non-iterative method for fitting dynamic paired comparison models. The method is especially useful when the population of objects or treatments to be compared

\footnotetext{
Address for correspondence: Mark E. Glickman, Department of Mathematics and Statistics, Boston University, 111 Cummington Street, Boston, MA 02215, USA.

E-mail: mg@math.bu.edu
} 
is large, and where parameter estimates are desired on an on-going basis. Measuring the abilities of chess players is the motivating example, though the method applies directly to other paired comparison settings where abilities or merits change over time. Section 2 introduces our dynamic paired comparison model. The non-iterative parameter updating algorithm is presented in Section 3. In our procedure, certain parameters need to be estimated before applying the updating algorithm, and the estimation of these model parameters is described in Section 4. The algorithm is then evaluated on simulated data in Section 5. Finally, the method is applied to two data sets: outcomes of chess games between the best chess players of all time and outcomes of tennis matches played between current tournament players.

\section{A dynamic paired comparison model}

The model that we assume for competitor ability is closely related to the Bradley-Terry model for paired comparisons (Bradley and Terry, 1952). The Bradley-Terry model asserts that, for two objects with merit parameters $\lambda_{1}$ and $\lambda_{2}$, object 1 is preferred to object 2 with probability $\lambda_{1} /\left(\lambda_{1}+\lambda_{2}\right)$. For our specific problem, let $\theta_{i}$ and $\theta_{j}$ be the unknown (scalar) strengths for players $i$ and $j$ at a fixed point in time. Assume first that a game results in only two outcomes: a win or a loss. Let $s_{i j k}$ be the $k$ th outcome of a game played between players $i$ and $j$, where $s_{i j k}=1$ when player $i$ wins and $s_{i j k}=0$ when player $j$ wins. The model for game outcomes, not allowing for ties or partial preferences, is given by

$$
\operatorname{Pr}\left(s_{i j k}=s\right)=\frac{\left(10^{\left(\theta_{i}-\theta_{j}\right) / 400}\right)^{s}}{1+10^{\left(\theta_{i}-\theta_{j}\right) / 400}},
$$

for $s=0,1$. This is simply a reparameterized version of the Bradley-Terry model. Likelihood-based inference for the Bradley-Terry model is straightforward from a set of paired comparison data. This particular reparameterization was chosen to produce parameter estimates that have an interpretation on the same scale as the USCF rating system, which corresponds to strength estimates roughly between 0 and 3000 .

Several extensions to the Bradley-Terry model incorporating ties have been proposed, including those by Davidson (1970) and Rao and Kupper (1967), each of whom introduced a single parameter governing the frequency of ties. Joe (1990) found that Davidson's model is not well fitted by a particular chess data set. In our likelihood framework, instead of adopting an approach that models a tie as a possible outcome of a game, we act as if ties do not really occur, but we treat a tie as half way between a win and a loss when constructing the likelihood. This approach avoids the complications of including extra (possibly time-varying) parameters in the model to account for the probability of a third outcome. More formally, we assume that the information about players' strengths resulting from a win followed by a loss is equivalent to the information resulting from two consecutive ties. Thus, if $p$ is the probability that the first player wins, so that the contribution to the likelihood of a win followed by a loss is $p(1-p)$, then the contribution to the likelihood of a single tie should be $\sqrt{ }\{p(1-p)\}$. We therefore construct a likelihood using terms in model (1) where a tie corresponds to $s=0.5$. Other extensions to the Bradley-Terry model, such as recognizing a home ground advantage for certain sports or the advantage of having the first move in chess, can be incorporated by the inclusion of an 'order effect' parameter, as in Davidson and Beaver (1977). We do not pursue this extension here.

Model (1) addresses measuring competitor ability when players' abilities remain fixed over 
time. This model can be extended to recognize that players' abilities can change over time. Glickman (1993) and Fahrmeir and Tutz (1994) explored an approach to modelling paired comparison data with time-varying abilities through the use of state space models. To use this approach, we assume that groups of comparisons considered to be collected in a short time interval are assumed to fall in the same 'rating period'. For example, chess tournament games played during a 2-month period could be considered to be part of the same rating period. Denoting the strength of player $i$ during a rating period at time $t_{0}$ by $\theta_{i}^{\left(t_{0}\right)}$, and the strength of player $i$ a rating period $t$ units of time later by $\theta_{i}^{\left(t_{0}+t\right)}$, we adopt a model that assumes

$$
\theta_{i}^{\left(t_{0}+t\right)} \mid \theta_{i}^{\left(t_{0}\right)}, \nu^{2}, t \sim N\left(\theta_{i}^{\left(t_{0}\right)}, \nu^{2} t\right),
$$

where $\nu^{2}$ is the increase in variance in competitors' strength per unit time. This model asserts that, as time passes while a player is not competing, the description of a competitor's strength becomes more uncertain. Knowledge of a player's activities (e.g. preparation) between events could be incorporated in model (2), but we assume that such information is not generally available.

A likelihood-based analysis of paired comparison data using state space models can follow either Fahrmeir and Tutz (1994), who used empirical Bayes methods, or Glickman (1993), who used Markov chain Monte Carlo simulation from the posterior distribution. In both approaches, a complete analysis involves estimating all the parameters jointly. With a small population of competitors (e.g. teams in a league), this does not present a difficulty. However, because competitions in many organizations involve populations of thousands of players (e.g. chess or interactive games on the Internet), an exact likelihood-based analysis may not be computationally feasible.

\section{An approximate Bayesian updating algorithm}

Suppressing the superscript $t$, let $\theta$ be the strength parameter of a player whose ability is to be estimated. Before a rating period, we assume that the prior distribution of a player's strength is

$$
\theta \mid \mu, \sigma^{2} \sim N\left(\mu, \sigma^{2}\right)
$$

with $\mu$ and $\sigma^{2}$ known. During a rating period, the player competes against $m$ opponents, playing $n_{j}$ games against opponent $j$, where $j=1, \ldots, m$. We assume that the distribution of the $j$ th opponent's strength, $\theta_{j}$, is

$$
\theta_{j} \mid \mu_{j}, \sigma_{j}^{2} \sim N\left(\mu_{j}, \sigma_{j}^{2}\right)
$$

with known $\mu_{j}$ and $\sigma_{j}^{2}$. Let $s_{j k}$ be the score of the $k$ th game against opponent $j$, with $s_{j k}=1$ if the player wins game $k, s_{j k}=0.5$ if the game results in a tie and $s_{j k}=0$ if the player loses. As before, we assume that the likelihood will be a product of Bradley-Terry 'probabilities'

$$
p\left(s_{j k} \mid \theta, \theta_{j}\right)=\frac{\left(10^{\left(\theta-\theta_{j}\right) / 400}\right)^{s_{j k}}}{1+10^{\left(\theta-\theta_{j}\right) / 400}} .
$$

The following sections develop a rating system based on closed form approximations to the computations required to perform a fully Bayesian model fit of the state space model described in Section 2. The rating algorithm is implemented as follows.

(a) Collect game outcome data over a rating period. 
(b) At the end of the period, update players' rating distributions due to game outcomes from their preperiod (prior) rating distributions.

(c) Subsequently update players' rating distributions due to the passage of time.

This is repeated for every rating period.

We proceed by describing prior distributions for players who had not previously competed in tournaments, then the procedure for computing posterior distributions of strengths due to the passage of time and finally the procedure for updating the strength distributions due to game results. The updating algorithm from game results is assessed in a simulation analysis. We also describe a smoothing procedure to make inferences on player strengths for early rating periods.

\subsection{Prior distribution of strengths}

Assume that, before competing, players' strengths are drawn independently from a normal distribution with mean 1500 and unknown variance. The initial variance $\sigma_{0}^{2}$ is treated as a parameter to be inferred from data. Thus, the prior distribution assumed for any player with strength parameter $\theta$ before competing is

$$
\begin{gathered}
\theta \mid \sigma_{0}^{2} \sim N\left(1500, \sigma_{0}^{2}\right), \\
\pi\left(\sigma_{0}^{2}\right) \propto 1 .
\end{gathered}
$$

Incorporating useful sources of information (such as players' ages) could result in more informative prior distributions, though for the development of the algorithm such information is assumed to be unavailable.

\subsection{Updating from the passage of time}

In performing the updating computations, we act as if all games in a rating period are played at the beginning of the period. Over the duration of the rating period, we assume that knowledge of a player's strength becomes less certain, so that the parameter variance increases. At time $t_{0}$, assume that a player's strength is distributed as

$$
\theta^{\left(t_{0}\right)} \mid \mu, \sigma^{2} \sim N\left(\mu, \sigma^{2}\right) .
$$

Integrating the distribution of $\theta^{\left(t_{0}+t\right)} \mid \theta^{\left(t_{0}\right)}, \nu^{2}, t \sim N\left(\theta^{\left(t_{0}\right)}, \nu^{2} t\right)$ with respect to the prior distribution (6) yields

$$
\theta^{\left(t_{0}+t\right)} \mid \mu, \sigma^{2}, \nu^{2}, t \sim N\left(\mu, \sigma^{2}+\nu^{2} t\right),
$$

where $\nu^{2}$ is the increase in variance per unit time. In other words, an elapse of $t$ units in time corresponds to an increase in $\nu^{2} t$ in the variance. This model for the increase in variance preserves the additivity of variance with respect to time. The variance per unit time, $\nu^{2}$, is a parameter that needs to be inferred in the model fitting process.

The choice of the length of a rating period involves a variance-bias trade-off. For short rating periods, few data may be available to estimate players' strengths, and the analytic approximations used in the algorithm in Section 3.3 may not be dependable. Conversely, if long rating periods are used, a player's ability may have changed substantially over a rating period, but this would not be detectable. The best compromise seems to be rating periods that are as short as possible, but where enough data are available to have some indication of players' strengths, perhaps at least 5-10 games per player on average. 


\subsection{Updating from outcomes of games}

At the beginning of a rating period, every competitor has a normal prior distribution of their playing strength. Rather than determine the posterior distribution of all strength parameters simultaneously making use of all paired comparison information, we carry out an approximate Bayesian analysis which leads to a tractable set of closed form computations. The key idea is that the marginal posterior distribution of a player's strength is determined by integrating out the opponents' strength parameters over their prior distribution rather than over their posterior distribution. The main disadvantage of this approach is that potentially important information is sacrificed, particularly the outcomes of opponents' games against other opponents. This is done to derive a set of closed form computations. Thus inferences from our algorithm will not be as precise as a fully Bayesian analysis. This sacrifice of precision appears necessary if posterior updates are needed on an on-going basis.

Letting $\mathbf{s}$ be the collection of outcomes of games during a rating period, the marginal posterior distribution of $\theta$ can be approximated as the integral of the posterior distribution of $\theta$ conditional on opponents' strengths integrated over their prior distribution,

$$
f(\theta \mid \mathbf{s}) \approx \int \ldots \int f\left(\theta \mid \theta_{1}, \ldots, \theta_{m}, \mathbf{s}\right) \varphi\left(\theta_{1} \mid \mu_{1}, \sigma_{1}^{2}\right) \ldots \varphi\left(\theta_{m} \mid \mu_{m}, \sigma_{m}^{2}\right) \mathrm{d} \theta_{1} \ldots \mathrm{d} \theta_{m},
$$

where $\varphi(\cdot)$ is the normal density with the given mean and variance, and

$$
f\left(\theta \mid \theta_{1}, \ldots, \theta_{m}, \mathbf{s}\right) \propto \varphi\left(\theta \mid \mu, \sigma^{2}\right) L\left(\theta, \theta_{1}, \ldots, \theta_{m} \mid \mathbf{s}\right) .
$$

Here, $L\left(\theta, \theta_{1}, \ldots, \theta_{m} \mid \mathbf{s}\right)$ is the likelihood for all parameters. As terms in the likelihood that do not depend on $\theta$ (which correspond to games played between other players) may be treated as constant with respect to $\theta$, the marginal posterior distribution of $\theta$ can be written as

$$
\begin{aligned}
f(\theta \mid \mathbf{s}) & \propto \int \ldots \int \varphi\left(\theta \mid \mu, \sigma^{2}\right) L\left(\theta, \theta_{1}, \ldots, \theta_{m} \mid \mathbf{s}\right) \varphi\left(\theta_{1} \mid \mu_{1}, \sigma_{1}^{2}\right) \ldots \varphi\left(\theta_{m} \mid \mu_{m}, \sigma_{m}^{2}\right) \mathrm{d} \theta_{1} \ldots \mathrm{d} \theta_{m} \\
& =\varphi\left(\theta \mid \mu, \sigma^{2}\right) \int \ldots \int L\left(\theta, \theta_{1}, \ldots, \theta_{m} \mid \mathbf{s}\right) \varphi\left(\theta_{1} \mid \mu_{1}, \sigma_{1}^{2}\right) \ldots \varphi\left(\theta_{m} \mid \mu_{m}, \sigma_{m}^{2}\right) \mathrm{d} \theta_{1} \ldots \mathrm{d} \theta_{m} \\
& \propto \varphi\left(\theta \mid \mu, \sigma^{2}\right) \prod_{j=1}^{m} \int \prod_{k=1}^{n_{j}} \frac{\left(10^{\left(\theta-\theta_{j}\right) / 400}\right)^{s_{j k}}}{1+10^{\left(\theta-\theta_{j}\right) / 400}} \varphi\left(\theta_{j} \mid \mu_{j}, \sigma_{j}^{2}\right) \mathrm{d} \theta_{j} .
\end{aligned}
$$

This expression may be evaluated by using numerical methods, such as Monte Carlo integration. Instead, we determine a set of closed form computations that approximate the marginal posterior density in expression (8). The details of the derivation are in Appendix A.

The updating algorithm approximates expression (8) by a normal density with mean and variance parameters $\mu^{\prime}$ and $\sigma^{\prime 2}$ respectively. The parameters are given by

$$
\begin{gathered}
\mu^{\prime}=\mu+\frac{q}{1 / \sigma^{2}+1 / \delta^{2}} \sum_{j=1}^{m} \sum_{k=1}^{n_{j}} g\left(\sigma_{j}^{2}\right)\left\{s_{j k}-E\left(s \mid \mu, \mu_{j}, \sigma_{j}^{2}\right)\right\}, \\
\sigma^{\prime 2}=\left(\frac{1}{\sigma^{2}}+\frac{1}{\delta^{2}}\right)^{-1}
\end{gathered}
$$

where 


$$
\begin{gathered}
q=\log (10) / 400=0.0057565, \\
g\left(\sigma^{2}\right)=\frac{1}{\sqrt{ }\left(1+3 q^{2} \sigma^{2} / \pi^{2}\right)}, \\
E\left(s \mid \mu, \mu_{j}, \sigma_{j}^{2}\right)=\frac{1}{1+10^{-g\left(\sigma_{j}^{2}\right)\left(\mu-\mu_{j}\right) / 400}}, \\
\delta^{2}=\left[q^{2} \sum_{j=1}^{m} n_{j} g\left(\sigma_{j}^{2}\right)^{2} E\left(s \mid \mu, \mu_{j}, \sigma_{j}^{2}\right)\left\{1-E\left(s \mid \mu, \mu_{j}, \sigma_{j}^{2}\right)\right\}\right]^{-1} .
\end{gathered}
$$

These calculations are carried out in parallel for each player individually over the rating period to produce the approximating normal posterior distributions of each player's strength.

The posterior mean updating approximation in equation (9) has close connections to the chess rating system of Elo (1978). Elo's system was adopted by the USCF in the early 1960s and subsequently by the World Chess Federation in 1970. Most national chess organizations, as well as national organizations for tournament table-tennis and Scrabble, have adopted Elo's system with minor variations. If a player has an estimated strength $\tilde{\theta}$ at the onset of a rating period, then the Elo algorithm for updating a player's strength estimate from the outcomes of games is given by

$$
\tilde{\theta}^{\prime}=\tilde{\theta}+K \sum_{j=1}^{m} \sum_{k=1}^{n_{j}}\left\{s_{j k}-\mathrm{We}\left(\tilde{\theta}, \tilde{\theta}_{j}\right)\right\},
$$

where $\tilde{\theta}^{\prime}$ is the player's posterior strength estimate, $\tilde{\theta}_{j}$ is the preperiod strength estimate of opponent $j, K$ is a constant (e.g. $K=32$ in the USCF system for amateur players) and

$$
\mathrm{We}\left(\tilde{\theta}, \tilde{\theta}_{j}\right)=\frac{1}{1+10^{-\left(\tilde{\theta}-\tilde{\theta}_{j}\right) / 400}}
$$

is the approximate probability of defeating player $j$ as a function of the estimates of strength.

The Elo updating algorithm is seen to be a special case of equation (9). If $\sigma_{j}^{2}=0$ for all opponents, implying that all opponents' mean strengths are known without error, then $g\left(\sigma_{j}^{2}\right)=1$ for all $j$, and $E\left(s \mid \mu, \mu_{j}, \sigma_{j}^{2}\right)=\mathrm{We}\left(\mu, \mu_{j}\right)$. Then, equation (9) reduces to the Elo updating formula with

$$
K=\frac{q}{1 / \delta^{2}+1 / \sigma^{2}}
$$

The computations in equations (9) and (10) may be preferable because they make use of the expected game outcome incorporating the uncertainty in the player's own strength and in the opponents' strengths, and the variability due to the game outcomes (represented by $\delta^{2}$ ). The updating formula (9) distinguishes the uncertainty in opponents' strengths by allowing $g(\cdot)$ to be less than 1; when an opponent's prior rating variance $\sigma_{j}^{2}$ is large, then $g\left(\sigma_{j}^{2}\right)$ is substantially less than 1, and therefore the contribution of this opponent to the sum in equation (9) will be relatively small. Also, the fraction in equation (13) in effect weights the results of games relative to the precision of one's strength before a competition. Thus, in contrast with the Elo updating formula, the value of $K$ depends on the prior precision of one's strength. When one's prior strength is uncertain, the outcomes of games have a potentially substantial effect on one's strength distribution update, and this is reflected in a large value of equation (13). When one's prior strength is precisely measured, then the outcomes of games should have little effect on one's strength update, and equation (13) is small. 


\subsection{Accuracy of approximation}

The accuracy of the approximation to the marginal posterior distribution (8) by a normal distribution with parameters given by equations (9) and (10) is assessed through simulation. We examine the accuracy of the updating algorithm when a player competes against 4, 10, 20 or 50 opponents in a rating period.

For a given number of opponents in a rating period, a player with prior strength distribution $\theta \sim N\left(1500,100^{2}\right)$ competes against opponents with normal prior distributions having means drawn from $N\left(1500,100^{2}\right)$ and standard deviations drawn from a scaled inverse $\chi^{2}$ distribution on 10 degrees of freedom with mean 50 . Outcomes of games were determined by simulating strengths from the prior distributions, and then simulating binary outcomes given the strengths. From a simulated collection of game outcomes and opponents' prior strength distributions, the computations in equations (9) and (10) were carried out. Additionally, 500 values of the marginal posterior distribution of $\theta$ given in expression (8) were simulated via Monte Carlo integration by using the weighted bootstrap (Smith and Gelfand, 1992). This was accomplished by generating 10000 random draws of $\theta$ from the player's prior distribution, determining the corresponding (approximate) marginal likelihoods evaluated at each of the draws (which involved averaging Bradley-Terry probabilities over 50 random draws from each opponent's prior distribution) and then drawing 500 values of $\theta$ without replacement from the original 10000 with probabilities proportional to the marginal likelihoods. The 500 values were summarized by means and standard deviations, and also by the proportion of values that lay within nominal $50 \%$ and $95 \%$ normal posterior intervals based on the approximating mean and standard deviation from equations (9) and (10). This entire process of calculating posterior summaries by the approximating method and the weighted bootstrap for each collection of simulated parameters and outcomes of games was repeated 500 times for each fixed number of opponents.

The results of the analyses are displayed in Table 1. The entries of Table 1 summarize the results of the 500 simulated data sets per number of opponents. On average, for 4, 10, 20 or 50 opponents in one rating period, approximately $50 \%$ and $95 \%$ of weighted bootstrap draws fall within $50 \%$ and $95 \%$ nominal posterior intervals constructed from the approximating normal distribution using the updating formulae. This suggests that, on average, the approximating normal distribution produced by the updating algorithm closely approximates the distribution of values determined from the weighted bootstrap. Because the entries in Table 1 are slightly less, on average, than the nominal coverage fractions, the updating algorithm produces mean and variance estimates that slightly overstate the precision of the marginal posterior distribution. One possible reason is that the updating algorithm produces

Table 1. Averages and $95 \%$ central intervals of the proportion of weighted bootstrap simulated values that fall within nominal $50 \%$ and $95 \%$ posterior intervals constructed from the approximating normal distribution $\dagger$

\begin{tabular}{|lcc|}
\hline \multirow{2}{*}{$\begin{array}{l}\text { Number of } \\
\text { opponents }\end{array}$} & \multicolumn{2}{c|}{ Average coverage fraction (95\% intervals) } \\
\cline { 2 - 3 } & Nominal 50\% posterior interval & Nominal 95\% posterior interval \\
\hline 4 & $0.4976(0.448,0.546)$ & $0.9480(0.929,0.967)$ \\
10 & $0.4959(0.449,0.536)$ & $0.9467(0.924,0.966)$ \\
20 & $0.4919(0.437,0.538)$ & $0.9452(0.922,0.966)$ \\
50 & $0.4873(0.433,0.538)$ & $0.9413(0.911,0.963)$ \\
\hline
\end{tabular}

$\dagger$ The coverage fractions and intervals were computed on the basis of 500 simulated data sets. 
posterior standard deviations that do not make full use of the data, resulting in variance estimates that are too small when a player has extreme results (relative to the prior distribution), and too large when a player's results are very consistent with the prior distribution. See Appendix A for computational details. This approximation becomes slightly amplified when more opponents are involved in the computations. It appears, however, that the approximating algorithm produces estimates that are sufficiently close to the Monte Carlo approach for practical purposes.

\subsection{Parameter smoothing}

The algorithm developed in Section 3.3 results in approximate posterior distributions of each player's strength parameter at the end of each rating period conditionally only on preceding outcomes of games. Thus, the algorithm makes efficient use of the data for estimating players' current strengths but makes poor use of the data for estimating strengths of players during early rating periods. A smoothing procedure is now described to obtain approximate posterior distributions of strength parameters about earlier rating periods conditionally on both previous and future data. This algorithm is the standard 'backward filtering' of the Kalman filter to obtain smoothed estimates of past parameters.

Let $t=1,2, \ldots, T$ sequentially index each of $T$ equally spaced rating periods. Denote the collection of all available outcomes of games in all rating periods before and including period $t$ by $\mathbf{s}^{(t)}$. Carrying out the (forward) rating algorithm, we obtain approximate normal posterior distributions of $\theta_{i}^{(t)} \mid \mathbf{s}^{(t)} \sim N\left(\mu_{i}^{(t)}, \sigma_{i}^{2(t)}\right)$, for $t=1, \ldots, T$ and for all players $i$, where $\theta_{i}^{(t)}$ denotes the strength parameter for player $i$ during rating period $t$. The posterior distribution of $\theta_{i}^{(T-1)} \mid \mathbf{s}^{(T)}$ can be determined by noting that

$$
f\left(\theta_{i}^{(T-1)} \mid \mathbf{s}^{(T)}\right) \propto \int f\left(\theta_{i}^{(T-1)} \mid \mathbf{s}^{(T-1)}\right) f\left(\theta_{i}^{(T)} \mid \theta_{i}^{(T-1)}, \nu^{2}\right) f\left(\theta_{i}^{(T)} \mid \mathbf{s}^{(T)}\right) \mathrm{d} \theta_{i}^{(T)} .
$$

Because all the densities in the integrand are assumed normal, the posterior distribution of $\theta_{i}^{(T-1)}$ is also normal, i.e. $\theta_{i}^{(T-1)} \mid \mathbf{s}^{(T)} \sim N(M, V)$ where

$$
\begin{aligned}
& V=\left(\frac{1}{\sigma_{i}^{2(T-1)}}+\frac{1}{\nu^{2}+\sigma_{i}^{2(T)}}\right)^{-1}, \\
& M=V\left(\frac{\mu_{i}^{(T-1)}}{\sigma_{i}^{2(T-1)}}+\frac{\mu_{i}^{(T)}}{\nu^{2}+\sigma_{i}^{2(T)}}\right) .
\end{aligned}
$$

This procedure is then applied recursively to $t=T-2, T-3, \ldots, 1$ to obtain all the posterior distributions of strength parameters for player $i$ conditionally on all the data. The same procedure is then applied to each player.

\section{Model fitting}

The algorithm in the preceding sections depends on knowing $\sigma_{0}^{2}$, the variance describing the initial uncertainty in players' abilities, and $\nu^{2}$, the variance increase per unit time. This section describes a procedure to estimate these parameters.

Let $t=1,2, \ldots, T$ index each of $T$ rating periods, $\mathbf{s}^{(t)}$ represent the collection of all available outcomes of games in all rating periods before and including period $t$, and $\theta_{i}^{(t)}$ and $\theta_{j}^{(t)}$ denote the strength parameters for players $i$ and $j$ during period $t$. For a given ordered 
pair of variances $\left(\sigma_{0}^{2}, \nu^{2}\right)$, the rating algorithm provides the computations to obtain the approximate distributions of $\theta_{i}^{(t)} \mid \mathbf{s}^{(t-1)} \sim N\left(\mu_{i}, \sigma_{i}^{2}\right)$ and $\theta_{j}^{(t)} \mid \mathbf{s}^{(t-1)} \sim N\left(\mu_{j}, \sigma_{j}^{2}\right)$. We may now define the 'predictive discrepancy' for a game between $i$ and $j$ during period $t$ as a measure of discrepancy between the predicted result for the game, given only information before period $t$, and the actual result of the game. In particular, let

$$
d_{i j}=-s_{i j} \log \left(p_{i j}\right)-\left(1-s_{i j}\right) \log \left(1-p_{i j}\right)
$$

be the discrepancy of a game played between $i$ and $j$, where $s_{i j}$ is the outcome of the game, and

$$
p_{i j}=\frac{1}{1+10^{-g\left(\sigma_{i}^{2}+\sigma_{j}^{2}\right)\left(\mu_{i}-\mu_{j}\right) / 400}} .
$$

Expression (16) is an approximation to the probability that $i$ defeats $j$ incorporating the variability of each player's strength estimate and is derived analogously to the expected outcome integrated over an opponent's prior strength distribution. Thus the predictive discrepancy $d_{i j}$ in equation (15) is the binomial log-likelihood for a game evaluated at a value that only depends on previous data.

The total discrepancy for the entire collection of outcomes of games is computed as the sum of the $d_{i j}$ over all games in the data set. This is accomplished by first summing the discrepancies in the first rating period, then updating the strength distributions based on the outcomes of games from the first period followed by updating the distributions due to the passage of time, then summing the discrepancies in the second rating period given the updated strength distributions, and so on. Because the discrepancies in results are cross-validatory, minimizing the total discrepancy is unlikely to result in an overfitted choice of variance parameters. Loss functions that involve measuring a discrepancy between predicted and actual outcomes, where the predicted outcome depends on all the data, may result in excessively large estimates of $\nu^{2}$ in order to allow a player's strengths to have unlimited variation from one rating period to the next.

Several numerical methods for minimizing the total discrepancy as a function of $\sigma^{2}$ and $\nu^{2}$ are feasible. However, because the total discrepancy is a complicated function of $\sigma^{2}$ and $\nu^{2}$, methods involving closed form derivatives are not possible. To minimize the total discrepancy, the Nelder-Mead simplex algorithm (Nelder and Mead, 1965) was employed. This algorithm uses a direct search approach rather than numerically computing gradient information.

\section{Evaluation of the estimation algorithm}

To evaluate the variance estimation procedure, the algorithm was applied to simulated data under three different sets of assumed parameters and conditions:

(a) simulation $1-10$ players competing over 30 rating periods, playing a total of 50 games per period, with $\sigma_{0}=200$ and $\nu=50$,

(b) simulation $2-10$ players competing over 120 rating periods, playing a total of 50 games per period, with $\sigma_{0}=200$ and $\nu=50$, and

(c) simulation 3-20 players competing over 50 rating periods, playing a total of 200 games per period, with $\sigma_{0}=200$ and $\nu=10$.

For each of the three simulation conditions, the fictitious competitors would compete with each other at random for the specified number of rating periods, over which their strengths 
Table 2. Results of simulation analyses $\uparrow$

\begin{tabular}{|c|c|c|c|c|c|c|}
\hline \multirow[t]{2}{*}{ Simulation } & \multicolumn{2}{|c|}{ Parameters } & \multicolumn{2}{|c|}{ Estimates } & \multicolumn{2}{|c|}{ Coverage fractions } \\
\hline & $\sigma_{0}$ & $\nu$ & $\hat{\sigma_{0}}$ & $\hat{\nu}$ & $50 \%$ & $95 \%$ \\
\hline 1 & 200 & 50 & 224.04 & 44.98 & 0.483 & 0.940 \\
\hline 2 & 200 & 50 & 240.10 & 44.64 & 0.446 & 0.912 \\
\hline 3 & 200 & 10 & 252.63 & 9.47 & 0.505 & 0.947 \\
\hline
\end{tabular}

† Outcomes of games were generated under differing model and data assumptions, and the table reports summaries over 200 replications. The second and third columns show the assumed variances that generated the simulated data. The estimates of the two variance parameters, which are shown in the fourth and fifth columns, are the averages over the 200 replications. The sixth and seventh columns show the proportion of $50 \%$ and $95 \%$ central posterior intervals that contain players' true mean strengths for the last rating period.

would change by the addition of $N\left(0, \nu^{2}\right)$ at the beginning of each successive rating period. This procedure was repeated 200 times for each of the three simulation conditions. From the results of each simulated data set, estimates of $\sigma_{0}$ and $\nu$ were obtained by using the approach in Section 4 and then used to compute ratings from the final rating period.

A summary of the analyses is shown in Table 2. The estimates of $\sigma_{0}$ and $\nu$ were calculated by using the simplex method, and the averages of these estimates over the 200 replications are shown in the fourth and fifth columns of Table 2 . The estimation procedure seems to produce estimates that are reasonably close to the underlying parameters. Sampling variability would explain some of the discrepancy. The slightly large estimates of $\sigma_{0}$ reflects that its posterior distribution is skewed. This can be seen in Fig. 1, which shows the approximate joint posterior distribution of the two standard deviation parameters. These plots demonstrate that the posterior distribution of the standard deviation parameters are centred near the generating parameters. The large posterior variability of $\sigma_{0}$ reflects the small number of players in the simulations. Not surprisingly, the posterior variability of $\nu$ is smaller when the number of rating periods is larger.

The last two columns of Table 2 show how well the estimated strengths from the last rating period correspond to the true parameter values. For each set of simulations, normal posterior distributions at the end of the last rating period are obtained for all players by using the estimated values of $\sigma_{0}$ and $\nu$. Also, the true mean strengths during the last rating period were recorded. After centring the estimated and true mean strengths to 1500 for each of the 200 replications, we determined the proportion of the players across the 200 replications that have $50 \%$ and $95 \%$ central posterior intervals containing their true mean strength at the last rating period. These appear in the last two columns in Table 2. The strengths were centred because drift in average strength cannot be detected from paired comparison data; if all players increased in strength by the same amount from one rating period to the next, it would be impossible to determine this from the data. In all cases, the actual proportion of coverage is close to the nominal level of coverage. This is particularly true for the third simulation which had parameters that were more precisely estimated because the size of the simulated data set per replication was large. For the first two simulations, the actual fraction of coverage is slightly less than the reported level of coverage, indicating that a player's approximate normal posterior distribution from the updating algorithm may be overstating the precision under similar conditions. However, the coverage is still fairly close to nominal, and with a large set of data coverage does not appear to be a problem. 


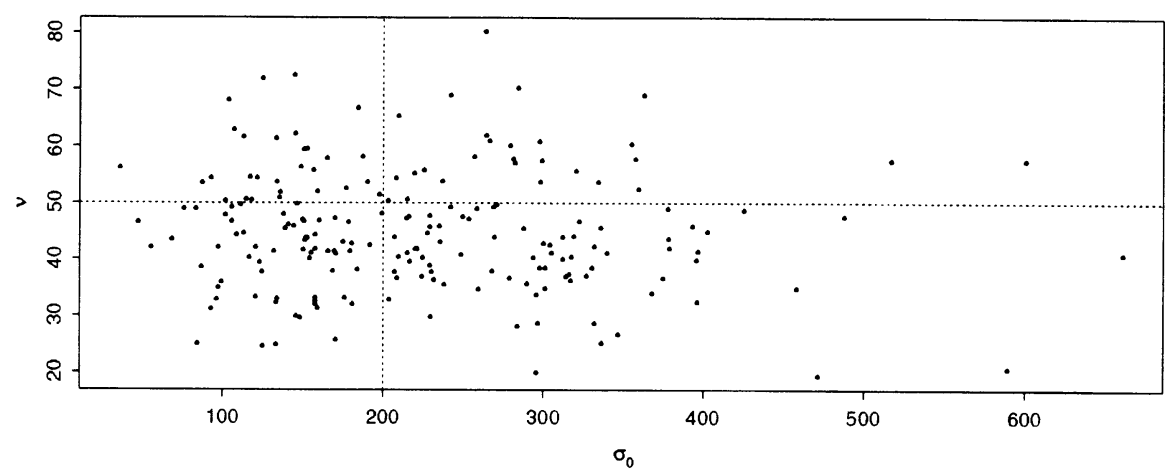

(a)

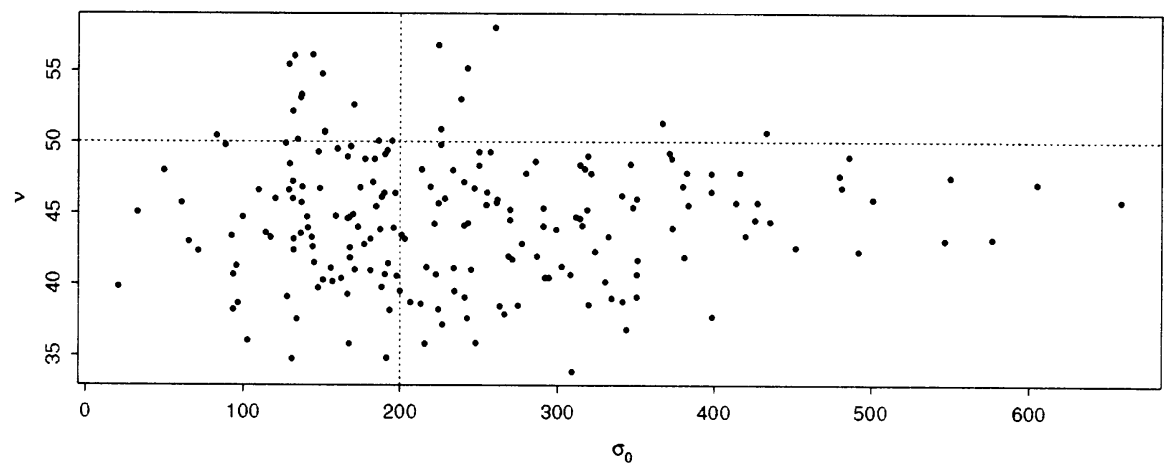

(b)

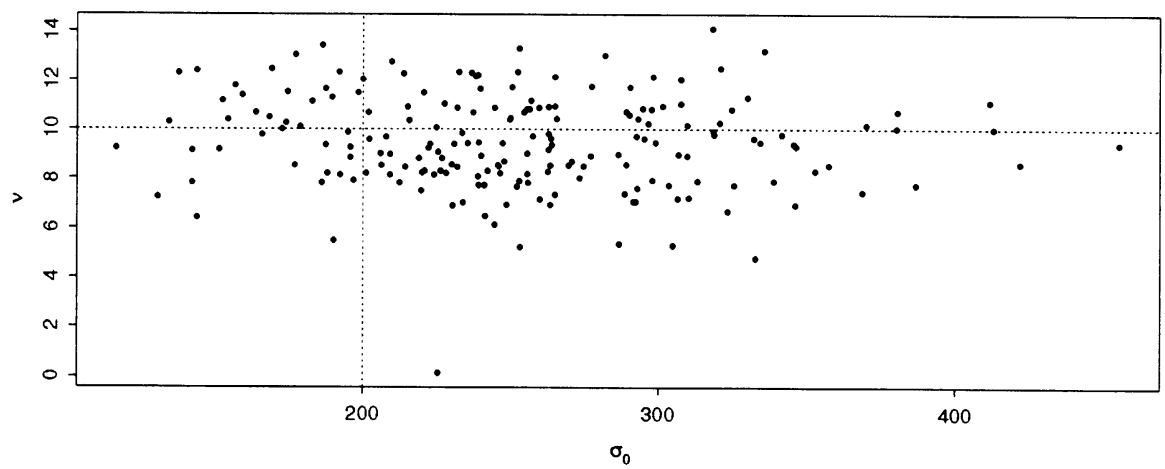

(c)

Fig. 1. Joint posterior distribution of $\left(\sigma_{0}, \nu\right)$ from (a) simulation 1, (b) simulation 2 and (c) simulation 3 : the vertical and horizontal dotted lines indicate the values of the generating parameters

\section{Examples}

We apply the methodology of Section 3 to the analysis of two data sets. The first data set consists of all known results of games from 1857 to 1991 between 88 of the world's all-time best chess players. The second data set involves the outcomes of tennis-matches played between 1190 competitors from 1986 to 1995. 


\subsection{The best chess players of all time}

Professor Nathan Divinsky has compiled a data set consisting of all known tournament or match game results between 88 of the top chess players of all time. The data set contains 15664 outcomes of games played between 1367 pairs of players. Not all $\left(\begin{array}{c}88 \\ 2\end{array}\right)=3828$ pairs of players competed against each other because some players' lives, much less their playing careers, did not overlap. Several analyses based on smaller versions of this data set have been published, including those by Elo (1978), Keene and Divinsky (1989), Joe (1990) and Henery (1992). Elo fitted paired comparison models separately in each year and then smoothed the estimates. Keene and Divinsky fitted a Bradley-Terry model to the data, acting as if all the games were played in one large tournament. Joe divided players' careers into 'peak' and 'offpeak' periods, and fitted an extension of the Bradley-Terry model explicitly accounting for ties. Henery fitted a model that accounts for differing frequencies of ties, but not the possibility of changes in abilities over time.

For our analysis, a single rating period is a year, so the number of rating periods is 135 . In some years no outcomes of games were observed (e.g. 1859, 1874 and 1875), but this is handled by applying the computations in Section 3.2 in succession. The prior variance for a player was constrained to be $\sigma^{2}$ for the year of first competition. The total discrepancy was minimized for $\sigma=38.19$ and $\nu=18.87$. Using these values, estimates of all players' strengths for every year were computed by using the rating algorithm followed by the backward smoothing procedure.

In Table 3, the top 20 players are ranked according to their peak posterior mean strength, along with the posterior standard deviation of the strength and the year in which the player attained highest strength (the peak year). The top eight players on the list are former and current world champions. The top eight players identified by Joe (1990) match the top eight

Table 3. 20 of the best chess players of all time ranked according to their posterior mean strength from the fitted model for the year in which the player attained highest strength, the peak year

\begin{tabular}{|clccc|}
\hline Rank & \multicolumn{1}{c}{ Player } & $\begin{array}{c}\text { Posterior mean } \\
\text { strength in peak } \\
\text { year }\end{array}$ & $\begin{array}{c}\text { Posterior } \\
\text { standard } \\
\text { deviation }\end{array}$ & $\begin{array}{c}\text { Peak } \\
\text { year }\end{array}$ \\
\hline 1 & Emanuel Lasker & 1693 & 29 & 1916 \\
2 & José Capablanca & 1680 & 28 & 1921 \\
3 & Robert Fischer & 1656 & 38 & 1972 \\
4 & Alexander Alekhine & 1647 & 24 & 1930 \\
5 & Garry Kasparov & 1643 & 32 & 1991 \\
6 & Mikhail Botvinnik & 1623 & 27 & 1947 \\
7 & Anatoly Karpov & 1609 & 20 & 1984 \\
8 & Wilhelm Steinitz & 1608 & 29 & 1876 \\
9 & Akiba Rubinstein & 1584 & 24 & 1912 \\
10 & Max Euwe & 1579 & 23 & 1935 \\
11 & Boris Spassky & 1578 & 21 & 1968 \\
12 & Siegbert Tarrasch & 1576 & 25 & 1905 \\
13 & Viktor Korchnoi & 1573 & 21 & 1978 \\
14 & Geza Maroczy & 1572 & 25 & 1908 \\
15 & David Bronstein & 1571 & 23 & 1953 \\
16 & Vassily Ivanchuk & 1570 & 32 & 1991 \\
17 & Samuel Reshevsky & 1569 & 24 & 1952 \\
18 & Vassily Smyslov & 1567 & 22 & 1954 \\
19 & Aron Nimzovitch & 1565 & 26 & 1931 \\
20 & Tigran Petrosian & 1564 & 22 & 1963 \\
\hline
\end{tabular}


in Table 3, except that Steinitz is replaced by Paul Morphy. The data set consists of only 25 games against two opponents played by Morphy between 1857 and 1858, so there is very little information in our analysis that would indicate such profound playing strength (Morphy is ranked 27 on our list). The ninth on the list, Akiba Rubinstein, is the highest ranked player who never held the world championship. Because of the possible increase in overall playing strength (which could result from an increased understanding of chess over time), the posterior mean strengths of players at different points in time cannot be directly compared. Instead, one should interpret Table 3 as indicating the level of strength relative to current competitors. Thus, according to the data analysis, Lasker dominated his opponents around 1916 more so than Fischer did in 1972.

Fig. 2 shows the strengths of the top eight players plotted over time. Typically, at the beginning of their careers, they are moderate in strength, with a gradual climb towards their peak strength and then a gradual decline. The most obvious exception is Fischer, who gave up competitive chess in 1972 after winning the world championship. The plot also suggests that Kasparov may have still been on the rise in 1991, and that Karpov has been gradually declining in strength since his peak in the mid-1980s. Both Alekhine and Botvinnik had careers that ended without a steep decline.

\subsection{Ranking current tennis-players}

The methodology of Section 3 was applied to rank the world's current top tennis-players. The data set consisted of all 33359 matches played between 1190 male participants from 1986 to

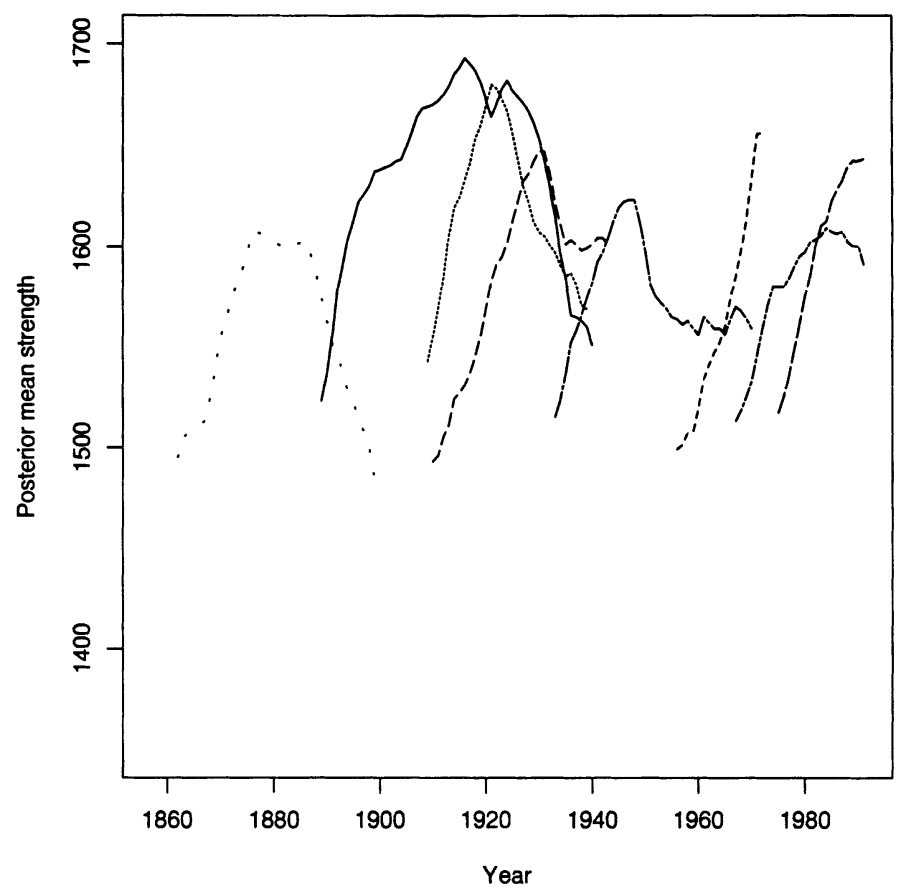

Fig. 2. Smoothed posterior mean strengths of eight players over time: . . . . , W. Steinitz; — —, E. Lasker; …...... J. Capablanca; - - -, A. Alekhine; — . - M. Botvinnik; - - - -, R. Fischer; - . - ., A. Karpov; ——, G. Kasparov 
the end of 1995 in events on the ATP tour. The ATP tour is generally regarded as the main international organizer of men's professional tennis. Games were excluded from the analysis if they were played in events that did not award ATP 'points', e.g. the Davis Cup and some ATP tour championships. Rating periods were designated to be 2 months long, resulting in 60 rating periods. As in the analysis of outcomes of chess games, the prior variance for a player was assumed to be $\sigma^{2}$ during the rating period in which he first competed.

Minimizing the total discrepancy for these data yielded values of $\sigma=113.65$ and $\nu=$ 22.35. In contrast with the chess outcomes analysis, the spread of players' initial strengths is much larger, indicating a wider variation of playing strengths among the tennis competitors. Because the chess players were selected to be (roughly) the 88 best in the world over all time, whereas the 1190 tennis-players were likely to be the best only in a 10-year period, the greater variability in the tennis-players' strengths is not surprising. The variance increase per year was much smaller for the chess player data than for the tennis data, suggesting more stability in chess playing strength than in tennis. This seems intuitively sensible because the factors that result in a tennis-player performing well (e.g. physical condition, responsiveness and lack of injuries) may be more variable over time than factors that result in a chess player performing well (judgment, insight and ability to calculate variations).

Table 4 displays the posterior means and standard deviations of the strengths for the top 20 players ranked according to their posterior means. Players were included in the list if they had played within four rating periods ( 8 months) of the end of 1995 . The posterior means for these top players have a greater spread than those for the top chess players, though because the standard deviations are also larger it is more difficult to assert differences in playing strength. Agassi and Sampras have posterior means that are about 100 higher than those for all other competitors. Posterior probabilities of game outcomes can be computed by using formula (16). According to the parameter estimates, Sampras, ranked second, would defeat Muster, ranked third, with a posterior probability of 0.63 .

Table 4. 20 of the current ATP tour tennis participants ranked according to their posterior mean strengths at the end of 1995 from the fitted model

\begin{tabular}{|rlccr|}
\hline Rank & \multicolumn{1}{c}{ Player } & $\begin{array}{c}\text { Posterior mean } \\
\text { strength }\end{array}$ & $\begin{array}{c}\text { Posterior standard } \\
\text { deviation }\end{array}$ & $\begin{array}{c}\text { ATP tour rank } \\
\text { at end of 1995 }\end{array}$ \\
\hline 1 & André Agassi & 1992 & 53 & 2 \\
2 & Pete Sampras & 1987 & 51 & 1 \\
3 & Thomas Muster & 1892 & 46 & 3 \\
4 & Michael Chang & 1885 & 50 & 5 \\
5 & Boris Becker & 1860 & 51 & 4 \\
6 & Jim Courier & 1841 & 48 & 8 \\
7 & Michael Stich & 1804 & 52 & 6 \\
8 & Yevgeny Kafelnikov & 1790 & 46 & 7 \\
9 & Thomas Enqvist & 1780 & 46 & 9 \\
10 & Wayne Ferreira & 1776 & 47 & 18 \\
11 & Todd Martin & 1767 & 50 & 17 \\
12 & Magnus Larsson & 1764 & 57 & 13 \\
13 & Sergi Bruguera & 1764 & 52 & 23 \\
14 & Goran Ivanisevic & 1757 & 51 & 11 \\
15 & Stefan Edberg & 1752 & 53 & 15 \\
16 & Richard Krajicek & 1747 & 40 & 14 \\
17 & Marc Rosset & 1720 & 43 & 16 \\
18 & Arnaud Boetsch & 1704 & 51 & 26 \\
19 & Andrei Medvedev & 1702 & 48 & \\
20 & Malivai Washington & 1695 & & \\
\hline
\end{tabular}


The last column of Table 4 shows the official ATP tour rankings of the players at the end of 1995 , based on players accruing points depending on their successes in their best 14 tournaments over the previous 12 months. The players are then ranked according to the sum of their points. Although this system does not use a probabilistic model to measure players' strengths, it does produce a ranking list that conforms to generally held perceptions. The top 18 players in the ATP tour rankings appear in Table 4. The other two players are Stefan Edberg, who is ranked 15 on our list, and Malivai Washington, who is ranked 20. Edberg appears high on our list because he was inferred to be much stronger in the early 1990s, and that the model fit purports that he did not decline in ability as much as the ATP ranking system indicates. Washington, interestingly, had the newsworthy result of competing in the finals at Wimbledon in 1996, so he may have been stronger, as our estimate shows, than indicated by the ATP rank.

\section{Discussion}

The problem of developing a rating system for large paired comparison experiments with time-varying abilities involves a trade-off between making full use of outcome information and keeping a system that is sufficiently simple to be used regularly. The likelihood-based state space approach provides inferences that are consistent with the model assumptions, but the computational complexity can be enormous. The algorithm developed in this paper attempts to combine desirable features of each approach to produce a system that is both usable and accurate for time-dependent paired comparison situations.

Although it is computationally straightforward, the algorithm ignores certain features of the model that a likelihood-based analysis would recognize. For example, information about the results of opponents' games is sacrificed to aid the computational ease of the algorithm. More precision could be gained in a likelihood-based analysis because all players' results would have an effect on inferences. A related issue is that posterior correlations of players' strengths are not accounted for in our algorithm. If a player competes against one particular opponent more frequently than against others, then a strong correlation may be induced. Then both strength distributions should be affected when either player competes. However, the benefit in excluding correlations is that the number of model parameters is greatly reduced. This is quite desirable when the number of players in the population is large. In any event, the simulations in this paper seem to show that the approximating algorithm performs reasonably well, and the application to the chess and tennis game data produced player rankings that match external criteria.

\section{Acknowledgements}

The author is grateful to Miki Singh and Harry Joe for providing the tennis data and chess data respectively and to Christopher Avery for his help in preparing the tennis data.

\section{Appendix A: Derivation of closed form computations}

We derive a closed form normal approximation to the approximate posterior distribution of a player's strength given in equation (8). The derivation of formulae (9) and (10) can be summarized in the following three steps.

(a) Approximate the likelihood, marginalized over the opponents' prior strength distribution, by a product of logistic cumulative distribution functions. 
(b) Approximate the resulting expression by a normal distribution.

(c) Construct a linear approximation by using a Taylor series expansion around the prior mean to determine the mean and variance of the approximating normal posterior distribution. by

The likelihood, integrated over the distribution of the opponents' prior strength distribution, is given

$$
\begin{aligned}
L(\theta \mid \mathbf{s}) & =\prod_{j=1}^{m} \int \prod_{k=1}^{n_{j}} \frac{\left(10^{\left(\theta-\theta_{j}\right) / 400}\right)^{s_{j k}}}{1+10^{\left(\theta-\theta_{j}\right) / 400}} \varphi\left(\theta_{j} \mid \mu_{j}, \sigma_{j}^{2}\right) \mathrm{d} \theta_{j} \\
& \approx \prod_{j=1}^{m} \prod_{k=1}^{n_{j}} \int \frac{\left(10^{\left(\theta-\theta_{j}\right) / 400}\right)^{s_{j k}}}{1+10^{\left(\theta-\theta_{j}\right) / 400}} \varphi\left(\theta_{j} \mid \mu_{j}, \sigma_{j}^{2}\right) \mathrm{d} \theta_{j} .
\end{aligned}
$$

Under the actual model, an opponent plays at a fixed strength (which can be viewed as a single value of $\theta_{j}$ drawn from the prior distribution) for all games in a rating period. This last approximation is justified by allowing the possibility that an opponent can display different strengths for different games, the strengths being drawn independently from the opponent's prior distribution.

An integral in the above product could be approximated easily by using numerical methods. Instead, we approximate the integrals in expression (17), which are logistic cumulative distribution functions (CDFs) integrated over normal densities, by rescaled logistic CDFs. This is accomplished by first approximating each logistic CDF in an integrand by a normal CDF with the same mean and variance so that the integral can be evaluated in closed form to a normal CDF. The resulting normal CDF is then converted back to a logistic CDF with the same mean and variance. This yields the approximation

$$
\int \frac{\left(10^{\left(\theta-\theta_{j}\right) / 400}\right)^{s_{j k}}}{1+10^{\left(\theta-\theta_{j}\right) / 400}} \varphi\left(\theta_{j} \mid \mu_{j}, \sigma_{j}^{2}\right) \mathrm{d} \theta_{j} \approx \frac{\left(10^{g\left(\sigma_{j}^{2}\right)\left(\theta-\mu_{j}\right) / 400}\right)^{s_{j k}}}{1+10^{g\left(\sigma_{j}^{2}\right)\left(\theta-\mu_{j}\right) / 400}},
$$

where

$$
g\left(\sigma^{2}\right)=\frac{1}{\sqrt{ }\left(1+3 q^{2} \sigma^{2} / \pi^{2}\right)}
$$

and

$$
q=\log (10) / 400 .
$$

Approximating integrals of this type, which commonly arise in logistic regression models with random effects, has been addressed similarly in Aitchison and Begg (1976), Lauder (1978) and Boys and Dunsmore (1987). The (approximate) marginal likelihood, therefore, may now be written as

$$
L(\theta \mid \mathbf{s})=\prod_{j=1}^{m} \prod_{k=1}^{n_{j}} \frac{\left(10^{g\left(\sigma_{j}^{2}\right)\left(\theta-\mu_{j}\right) / 400}\right)^{s_{j k}}}{1+10^{g\left(\sigma_{j}^{2}\right)\left(\theta-\mu_{j}\right) / 400}} .
$$

We now obtain a normal approximation to this marginal likelihood. To do so, we first find an expression for the mode by setting the derivative of the log-marginal likelihood equal to 0 . Note that

$$
\log \{L(\theta \mid \mathbf{s})\}=\sum_{j=1}^{m} \sum_{k=1}^{n_{j}}\left\{q g\left(\sigma_{j}^{2}\right) s_{j k}\left(\theta-\mu_{j}\right)-\log \left(1+10^{g\left(\sigma_{j}^{2}\right)\left(\theta-\mu_{j}\right) / 400}\right)\right\}
$$

so

$$
\frac{\partial[\log \{L(\theta \mid \mathbf{s})\}]}{\partial \theta}=q \sum_{j=1}^{m} \sum_{k=1}^{n_{j}} g\left(\sigma_{j}^{2}\right)\left(s_{j k}-\frac{1}{1+10^{-g\left(\sigma_{j}^{2}\right)\left(\theta-\mu_{j}\right) / 400}}\right) .
$$

Let

$$
E\left(s \mid \theta, \mu_{j}, \sigma_{j}^{2}\right)=\frac{1}{1+10^{-g\left(\sigma_{j}^{2}\right)\left(\theta-\mu_{j}\right) / 400}},
$$


which is the approximate expected outcome of a game against opponent $j$, incorporating the uncertainty contained in the prior distribution of this opponent's strength. At the mode of the marginal likelihood $\hat{\theta}$, we therefore have

$$
\sum_{j=1}^{m} \sum_{k=1}^{n_{j}} g\left(\sigma_{j}^{2}\right)\left\{s_{j k}-E\left(s \mid \hat{\theta}, \mu_{j}, \sigma_{j}^{2}\right)\right\}=0 .
$$

The second derivative of the log-marginal likelihood, evaluated at $\hat{\theta}$, is given by

$$
\begin{aligned}
\left.\frac{\partial^{2}[\log \{L(\theta \mid \mathbf{s})\}]}{\partial \theta^{2}}\right|_{\theta=\hat{\theta}} & =-q^{2} \sum_{j=1}^{m} \sum_{k=1}^{n_{j}} g\left(\sigma_{j}^{2}\right)^{2} E\left(s \mid \hat{\theta}, \mu_{j}, \sigma_{j}^{2}\right)\left\{1-E\left(s \mid \hat{\theta}, \mu_{j}, \sigma_{j}^{2}\right)\right\} \\
& =-q^{2} \sum_{j=1}^{m} n_{j} g\left(\sigma_{j}^{2}\right)^{2} E\left(s \mid \hat{\theta}, \mu_{j}, \sigma_{j}^{2}\right)\left\{1-E\left(s \mid \hat{\theta}, \mu_{j}, \sigma_{j}^{2}\right)\right\} .
\end{aligned}
$$

The marginal likelihood can be approximated by a normal density with a mean equal to $\hat{\theta}$ and a variance which is the negative reciprocal of expression (22). Let $\delta^{2}$ be the variance associated with the normal approximation to the marginal likelihood. We can effectively approximate $\delta^{2}$ by substituting $\mu$, the prior mean, for $\hat{\theta}$,

$$
\delta^{2} \approx\left[q^{2} \sum_{j=1}^{m} n_{j} g\left(\sigma_{j}^{2}\right)^{2} E\left(s \mid \mu, \mu_{j}, \sigma_{j}^{2}\right)\left\{1-E\left(s \mid \mu, \mu_{j}, \sigma_{j}^{2}\right)\right\}\right]^{-1},
$$

because the binomial-type variance in equation (22) is roughly constant over a wide range of values of $\theta$.

The approximate marginal posterior distribution for $\theta$ can now be expressed as proportional to a product of two normal densities,

$$
f(\theta \mid \mathbf{s}) \propto \varphi\left(\theta \mid \mu, \sigma^{2}\right) \varphi\left(\theta \mid \hat{\theta}, \delta^{2}\right) .
$$

The posterior mean $\mu^{\prime}$ and posterior variance $\sigma^{\prime 2}$ can therefore be expressed as

$$
\begin{aligned}
& \sigma^{\prime 2}=\left(1 / \sigma^{2}+1 / \delta^{2}\right)^{-1}, \\
\mu^{\prime}= & \sigma^{\prime 2}\left(\mu / \sigma^{2}+\hat{\theta} / \delta^{2}\right) \\
= & \mu+\frac{1 / \delta^{2}}{1 / \sigma^{2}+1 / \delta^{2}}(\hat{\theta}-\mu) .
\end{aligned}
$$

Rather than calculating $\hat{\theta}$ directly by using an iterative numerical procedure, we use a linear closed form approximation to $\hat{\theta}-\mu$. Define

$$
h(\theta)=\sum_{j=1}^{m} \sum_{k=1}^{n_{j}} \frac{g\left(\sigma_{j}^{2}\right)}{1+10^{-g\left(\sigma_{j}^{2}\right)\left(\theta-\mu_{j}\right) / 400}} .
$$

From equation (21), we now have that

$$
h(\hat{\theta})=\sum_{j=1}^{m} \sum_{k=1}^{n_{j}} g\left(\sigma_{j}^{2}\right) s_{j k} .
$$

A Taylor series expansion of $h(\theta)$ around $\mu$ yields

$$
h(\hat{\theta}) \approx h(\mu)+(\hat{\theta}-\mu) h^{\prime}(\mu)
$$

where

$$
h^{\prime}(\mu)=q \sum_{j=1}^{m} \sum_{k=1}^{n_{j}} g\left(\sigma_{j}^{2}\right)^{2} E\left(s \mid \mu, \mu_{j}, \sigma_{j}^{2}\right)\left\{1-E\left(s \mid \mu, \mu_{j}, \sigma_{j}^{2}\right)\right\} .
$$

Substituting the linear approximation implied by approximation (29) for $\hat{\theta}-\mu$, equation (26) can be rewritten as 


$$
\begin{aligned}
\mu^{\prime} & \approx \mu+\frac{1 / \delta^{2}}{1 / \sigma^{2}+1 / \delta^{2}} \frac{h(\hat{\theta})-h(\mu)}{h^{\prime}(\mu)} \\
& =\mu+\frac{q}{1 / \sigma^{2}+1 / \delta^{2}}\{h(\hat{\theta})-h(\mu)\} \\
& =\mu+\frac{q}{1 / \sigma^{2}+1 / \delta^{2}} \sum_{j=1}^{m} \sum_{k=1}^{n_{j}} g\left(\sigma_{j}^{2}\right)\left\{s_{j k}-E\left(s \mid \mu, \mu_{j}, \sigma_{j}^{2}\right)\right\} .
\end{aligned}
$$

The equality in expression (31) follows as $1 / \delta^{2} h^{\prime}(\mu)=q$. The last equality in expression (32) is justified by expanding the expressions for $h(\hat{\theta})$ and $h(\mu)$. The posterior mean and variance, therefore, can be computed by using closed form equations (25) and (32).

\section{References}

Aitchison, J. and Begg, C. B. (1976) Statistical diagnosis when basic cases are not classified with certainty. Biometrika, 63, 1-12.

Boys, R. J. and Dunsmore, I. R. (1987) Diagnostic and sampling models in screening. Biometrika, 74, $365-374$.

Bradley, R. A. (1984) Paired comparisons: some basic procedures and examples. In Handbook of Statistics (eds P. R. Krishnaiah and P. K. Sen), vol. 4, pp. 299-326. Amsterdam: Elsevier.

Bradley, R. A. and Terry, M. E. (1952) The rank analysis of incomplete block designs: 1, The method of paired comparisons. Biometrika, 39, 324-345.

David, H. A. (1988) The Method of Paired Comparisons, 2nd edn. London: Chapman and Hall.

Davidson, R. R. (1970) On extending the Bradley-Terry model to accommodate ties in paired comparison experiments. J. Am. Statist. Ass., 65, 317-328.

Davidson, R. R. and Beaver, R. J. (1977) On extending the Bradley-Terry model to incorporate within-pair order effects. Biometrics, 33, 693-702.

Elo, A. E. (1978) The Rating of Chess Players Past and Present. New York: Arco.

Fahrmeir, L. and Tutz, G. (1994) Dynamic stochastic models for time-dependent ordered paired comparison systems. J. Am. Statist. Ass., 89, 1438-1449.

Glickman, M. E. (1993) Paired comparison models with time-varying parameters. PhD Dissertation. Department of Statistics, Harvard University, Cambridge.

Henery, R. J. (1992) An extension to the Thurstone-Mosteller model for chess. Statistician, 41, 559-567.

Joe, H. (1990) Extended use of paired comparison models, with application to chess rankings. Appl. Statist., 39, 85-93.

Keene, R. and Divinsky, N. (1989) Warriors of the Mind: a Quest for the Supreme Genius of the Chess Board. Brighton: Hardinge Simpole.

Lauder, I. J. (1978) Computational problems in predictive diagnosis. In COMPSTAT: Proc. 3rd Symp. Computational Statistics, Leiden (eds L. C. A. Corsten and J. Hermans), pp. 186-192. Leiden: Physica.

Nelder, J. A. and Mead, R. (1965) A simplex method for function minimization. Comput. J., 7, 308-313.

Rao, P. V. and Kupper, L. L. (1967) Ties in paired-comparison experiments: a generalization of the Bradley-Terry model. J. Am. Statist. Ass., 62, 194-204.

Smith, A. F. M. and Gelfand, A. E. (1992) Bayesian statistics without tears: a sampling-resampling perspective. $A m$. Statistn, 46, 84-88. 